Int. Arch. Allergy 1960;17(suppl 1):I-VI

\title{
Contents, Supplement 1, 1960
}

\section{Index}

Einleitung und Problemstellung

I. Die Häufigkeitsverhältnisse

A. Bisherige Untersuchungen

B. Eigene Untersuchungen

Disposition und Vorbereitung der Populationsstatistik

Methodik der medizinischen und statistischen Auswertung ...

Material

11

Alter und Geschlecht der Antwortenden

Häufigkeit von Asthma bronchiale resp. asthmoider Bronchitis, Rhinitis atopica und

Neurodermitis

12

Häufigkeiten der einzelnen atopischen Krankheiten und ihrer Kom-binationen

Häufigkeit der Genotypen und Genfrequenz für die Erbgangshypo-thesen Rezessivität und

Dominanz 18

II. Die zwillingspathologischen Verhältnisse
A.
Bisherige Untersuchungen
23

B. Eigene Untersuchungen

Materialgewinnung

24

Zwillingsdiagnostik

24

Prüfung auf Interessantheitsauslese

Kasuistik

$$
25
$$

Diskussion der Ergebnisse

III. Die familienpathologischen Verhältnisse

A. Bisherige Untersuchungen 28

B. Eigene Untersuchungen 35

Material 35

Methodik 37

Prüfung auf genetische Zusammengehörigkeit der Atopien und der Allergien im weiteren Sinn 38

Das korrelative Verhalten der Atopien und der «minor allergies» 40

Häufigkeit von Urticaria, Kontaktekzem und Medikamentenüber-empfindlichkeiten bei

Atopikern 44

Geschlechtsverteilung der Atopien

46

Das Manifestationsalter und seine Beziehungen zur familiären Be-lastung

Die phänotypischen Belastungsverhältnisse $\quad 50$

a) Definition der phänotypischen Belastung $\quad 50$

Belastungsverhältnisse der Neurodermitiker $\quad 50$

Belastungsverhältnisse der Asthma-Rhinitiker (Respirations-atopiker) 52

Belastungsverhältnisse der «hauttestpositiven» und «hauttest-negativen» Atopiker 
9. Das intrafamiliäre Verhalten der Reaginbildung

10. Die Erbgangsfrage

IV. Körperhabitus und Atopie

A. Einleitung und bisherige Untersuchungen

B. Eigene Untersuchungen

Problemstellung 75

Methodik der Habitusdiagnostik

Größe und Altersverteilung des Materials

Die Habitusverhältnisse der Atopiker und Atopie-Konduktoren .

V. Die biologische Bedeutung der Ergebnisse

VI. Zusammenfassung.

VII. Stammbaumdokumentation

VIII. Literaturverzeichnis

Vorwort

Die allergischen Krankheiten der «Atopie», d. h. Neuroder-mitis, Kinderekzem, Asthma und

Rhinitis atopica, stellen noch viele ungelöste Probleme hinsichtlich Pathogenese, spezifischer

Diagnostik und Therapie. Die vorliegende Arbeit von U. W. Schnyder erzielte durch

gruppenmedizi ische Untersuchungen inter-essante Resultate.

Aus einer Bevölkerungsstatistik der Stadt Zurich konnte das relativ häufige Vorkommen der

Rhinitis atopica, das weniger häu-fige Auftreten von Asthma bronchiale und das relativ seltene der Neurodermitis disseminata erwiesen werden. Es zeigte sich, daß diese Krankheitsbilder einzeln oder kombiniert miteinander in Be-ziehung stehen. Die Bedeutung der erblichen Disposition ließ sich an der überwiegenden Konkordanz der Erkrankungen eineiiger Zwillinge erweisen. Die familienpathologischen Untersuchungen zeigten zudem einwandfrei, daß medikamentöse Allergie, Kontakt-ekzem und Urticaria bei Atopikern nicht häufiger auftreten als bei Gesunden, was neben den bereits bekannten allergologischen Be-sonderheiten dieser Krankheiten erneut auf einen anderen pathoge-netischen Mechanismus hindeutet.

Das Vorkommen von Hautreaginen bei den atopischen Affek-tionen scheint von erblichen Faktoren abhängig zu sein, indem bei eineiigen Zwillingen Hautpositivität oder -negativität parallel gingen und in den meisten Familien Erkrankte vorwiegend positive oder negative Hautreagibilität aufwiesen. Diese Tatsache stützt die schon lange geäußerte Vermutung, daß das Vorhandensein von Hautreaginen für die Entstehung der Krankheitsbilder nicht obligat ist. Das Befallensein von Haut, Nasenschleimhaut oder Bronchien, d. h. die Wahl des «Schockorgans», ist ebenfalls weitgehend von erblichen Faktoren abhängig. Bei der in der Arbeit nachgegangenen Hypothese einer unregelmäßig autosomal-dominanten Ver-

erbung der Atopien scheint nun aber doch eine Erkrankung nicht schicksalsmäßig auftreten zu müssen, indem ein großer Spielraum der Manifestation (Penetranz) durch wechselnde Urnweltsfaktoren und Lebensgewohnheiten bestehen bleibt. Ob nun aber andererseits bei fehlender erblicher Disposition der Mensch keinesfalls an atopischen Leiden erkranken kann, sollte erst experimentell bewiesen werden. Bei dem notwendigen Vorkommen von Solitärfällen bei unregelmäßiger Dominanz ist allerdings das Fehlen einer latenten Disposition nur schwer sicherzustellen.

Die konstitutionspathologischen Untersuchungen ergaben das überraschende Resultat, daß die 21-40jährigen Atopiker vorwie-gend leptosomer und myoser Konstitution sind, die 4160jährigen Atopiker hingegen habitusmäßig meistens zum pyknischen Formen-kreis gehören, genau so wie die genotypisch belasteten, aber phaeno-typisch gesunden Atopiekonduktoren 
dieser Altersklasse. Dies zeigt einmal mehr, wie notwendig die Berücksichtigung des Alters bei statistischen Untersuchungen ist, um Fehlschlüsse zu vermeiden.

Die interessanten und statistisch gesicherten Untersuchungen Schnyders weisen auf die außerordentliche Bedeutung der erblichen Disposition für die Pathogenese der atopischen Krankheiten hin, was mit neueren Untersuchungen der Klinik über die beson-dere Einstellung des vegetativen Nervensystems bei Atopikern übereinstimmt.

Die vorliegende Arbeit soil nicht nur einen Beitrag zum Problem der Atopien darstellen, sondern das biologische Verständnis für die erblichen Dispositionskrankheiten überhaupt fördern, deren Wesen allzu oft nur unter dem Gesichtspunkt des exogenen aetio-logischen Prinzipes betrachtet wird.

Zurich, im Juli 1960

H. Storck 